\title{
Transcriptional Profiling of the Candida auris Response to Exogenous Farnesol Exposure
}

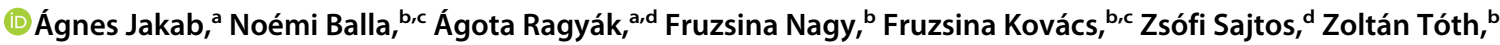 \\ (D) Andrew M. Borman, ${ }^{\text {e,f }}$ István Pócsi, ${ }^{a}$ Edina Baranyai, ${ }^{d}$ László Majoros, ${ }^{b}$ (Denátó Kovács ${ }^{\text {b,g }}$
}

\begin{abstract}
aDepartment of Molecular Biotechnology and Microbiology, Institute of Biotechnology, Faculty of Science and Technology, University of Debrecen, Debrecen, Hungary bDepartment of Medical Microbiology, Faculty of Medicine, University of Debrecen, Debrecen, Hungary

'Doctoral School of Pharmaceutical Sciences, University of Debrecen, Debrecen, Hungary

dDepartment of Inorganic and Analytical Chemistry, Agilent Atomic Spectroscopy Partner Laboratory, University of Debrecen, Debrecen, Hungary

eUK National Mycology Reference Laboratory, Public Health England, Science Quarter, Southmead Hospital, Bristol, United Kingdom

fMedical Research Council Centre for Medical Mycology (MRC CMM), University of Exeter, Exeter, United Kingdom

gFaculty of Pharmacy, University of Debrecen, Debrecen, Hungary
\end{abstract}

ABSTRACT The antifungal resistance threat posed by Candida auris necessitates bold and innovative therapeutic options. Farnesol is a quorum-sensing molecule with a potential antifungal and/or adjuvant effect; it may be a promising candidate in alternative treatment regimens. To gain further insights into the farnesol-related effect on C. auris, genome-wide gene transcription analysis was performed using transcriptome sequencing (RNA-Seq). Farnesol exposure resulted in 1,766 differentially expressed genes. Of these genes, 447 and 304 genes with at least 1.5-fold increase or decrease in transcription, respectively, were selected for further investigation. Genes involved in morphogenesis, biofilm events (maturation and dispersion), gluconeogenesis, iron metabolism, and regulation of RNA biosynthesis showed downregulation, whereas those related to antioxidative defense, transmembrane transport, glyoxylate cycle, fatty acid $\beta$-oxidation, and peroxisome processes were upregulated. In addition, farnesol treatment increased the transcription of certain efflux pump genes, including MDR1, CDR1, and CDR2. Growth, measured by the change in the number of CFU, was significantly inhibited within $2 \mathrm{~h}$ of the addition of farnesol $\left(5.8 \times 10^{7} \pm 1.1 \times 10^{7}\right.$ and $1.1 \times 10^{7} \pm$ $0.3 \times 10^{7} \mathrm{CFU} / \mathrm{ml}$ for untreated control and farnesol-exposed cells, respectively) $(P<$ $0.001)$. In addition, farnesol treatment caused a significant reduction in intracellular iron $(152.2 \pm 21.1$ versus $116.0 \pm 10.0 \mathrm{mg} / \mathrm{kg})$, manganese $(67.9 \pm 5.1$ versus $18.6 \pm 1.8 \mathrm{mg} /$ $\mathrm{kg})$, and zinc (787.8 \pm 22.2 versus $245.8 \pm 34.4 \mathrm{mg} / \mathrm{kg})(P<0.05$ to 0.001$)$ compared to untreated control cells, whereas the level of cooper was significantly increased $(274.6 \pm 15.7$ versus $828.8 \pm 106.4 \mathrm{mg} / \mathrm{kg})(P<0.001)$. Our data demonstrate that farnesol significantly influences the growth, intracellular metal ion contents, and gene transcription related to fatty acid metabolism, which could open new directions in developing alternative therapies against $C$. auris.

IMPORTANCE Candida auris is a dangerous fungal pathogen that causes outbreaks in health care facilities, with infections associated with a high mortality rate. As conventional antifungal drugs have limited effects against the majority of clinical isolates, new and innovative therapies are urgently needed. Farnesol is a key regulator molecule of fungal morphogenesis, inducing phenotypic adaptations and influencing biofilm formation as well as virulence. Alongside these physiological modulations, it has a potent antifungal effect alone or in combination with traditional antifungals, especially at supraphysiological concentrations. However, our knowledge about the mechanisms underlying this antifungal effect against $C$. auris is limited. This study has demonstrated that farnesol enhances the oxidative stress and reduces the fungal survival strategies. Furthermore, it inhibits manganese, zinc transport, and iron
Citation Jakab Á, Balla N, Ragyák Á, Nagy F, Kovács F, Sajtos Z, Tóth Z, Borman AM, Pócsi I, Baranyai E, Majoros L, Kovács R. 2021.

Transcriptional profiling of the Candida auris response to exogenous farnesol exposure. mSphere 6:e00710-21. https://doi.org/10.1128/ mSphere.00710-21.

Editor Aaron P. Mitchell, University of Georgia Copyright $\odot 2021$ Jakab et al. This is an openaccess article distributed under the terms of the Creative Commons Attribution 4.0 International license. Address correspondence to Renátó Kovács, kovacs.renato@med.unideb.hu.

Received 22 August 2021

Accepted 29 September 2021

Published 13 October 2021 
metabolism as well as increases fungal intracellular copper content. In addition, metabolism was modulated toward $\beta$-oxidation. These results provide definitive explanations for the observed antifungal effects.

KEYWORDS Candida auris, farnesol, quorum sensing, transcriptome analysis, oxidative stress, metal, iron, zinc, copper

dramatic increase in resistance to conventional antifungal agents has been reported for Candida auris worldwide, leading to evasion from efficient therapeutic options. The current coronavirus disease 2019 (COVID-19) pandemic situation may further promote the spreading of this fungal superbug. Superinfections by $C$. auris in critically ill COVID-19 patients have been related to high 30-day mortality rates, usually above $50 \%$ (1-3).

Farnesol is a fungal quorum-sensing molecule inducing hypha-yeast morphological switching in Candida albicans (4). In the past decade, several studies have reported that farnesol can generate oxidative stress and influence membrane permeability and cellular polarization in certain fungal species, especially at supraphysiological concentrations (57). Although farnesol does not affect the growth rate of $C$. albicans growing in the planktonic form, it significantly decreased the growth of $C$. auris regarding both planktonic cells and also 1-day-old biofilms of this organism (7). Recently, alternative therapeutic approaches designed to disturb quorum sensing have become an attractive treatment strategy $(8,9)$. The usage of farnesol and traditional antifungal drugs in combination may provide new insights into the management of newly emerged fungal species, such as $C$. auris, which poses a global threat to the nosocomial environment $(7,10)$.

Different metal ions facilitate numerous essential molecular processes within bacterial and fungal pathogens in quorum sensing-related pathways (11-13). Metals play a pivotal role in infection as cofactors in several enzymes related to metabolic activity and virulence, such as metal-dependent superoxide dismutases, metalloproteases, or melanin-producing laccases (14). We hypothesize that therapies interfering with quorum sensing may disturb the intracellular ion homeostasis, which may further elucidate the observed supraphysiological quorum-sensing molecule-related antifungal effect.

Previously, our group has reported the potential therapeutic benefit of farnesol against $C$. auris $(7,10)$. However, to date, there are no data that describe the total transcriptome changes induced by farnesol. Such data might help reveal the C. auris-specific response to exogenous farnesol exposure. To gain further insights into previously described physiological consequences of farnesol treatment, we determined genomewide gene transcription changes induced by farnesol exposure using total transcriptome sequencing (RNA-Seq).

\section{RESULTS}

Farnesol exposure inhibits the growth of Candida auris. The growth of $C$. auris was examined following $75 \mu \mathrm{M}$ farnesol treatment in yeast extract-peptone-dextrose (YPD). Adding farnesol to preincubated cells resulted in a remarkable growth inhibition, starting at $6 \mathrm{~h}$ postinoculation, which was confirmed by both absorbance (optical density at $\left.640 \mathrm{~nm}\left[\mathrm{OD}_{640}\right]\right)$ measurements and CFU determination. Growth was significantly inhibited within $2 \mathrm{~h}$ of the addition of farnesol as assessed both by CFU changes $\left(5.8 \times 10^{7} \pm\right.$ $1.1 \times 10^{7}$ and $1.1 \times 10^{7} \pm 0.3 \times 10^{7} \mathrm{CFU} / \mathrm{ml}$ for untreated control and farnesol-exposed cells, respectively) $(P<0.001)$ and observed absorbance values $(1.28 \pm 0.04$ and $0.72 \pm 0.04$ for untreated control and farnesol-exposed cells, respectively, at $\left.\mathrm{OD}_{640}\right)(P<$ 0.001 ) (Fig. 1). The observed growth inhibition was further confirmed by changes in measured dry cell mass (DCM) at 12-h incubation time $(5.5 \pm 0.2$ and $1.3 \pm 0.1 \mathrm{~g} /$ liter for untreated control and farnesol-exposed cells, respectively) $(P<0.001)$.

The ratios of nonviable cells were $3.3 \% \pm 1.2 \%, 1 \% \pm 0 \%, 1.7 \% \pm 0.6 \%$, and $3.6 \% \pm$ $0.6 \%$ for 0 -h cells, 4 -h cells, 6-h untreated and 6-h farnesol-exposed cells, respectively (Fig. 2A to D). 


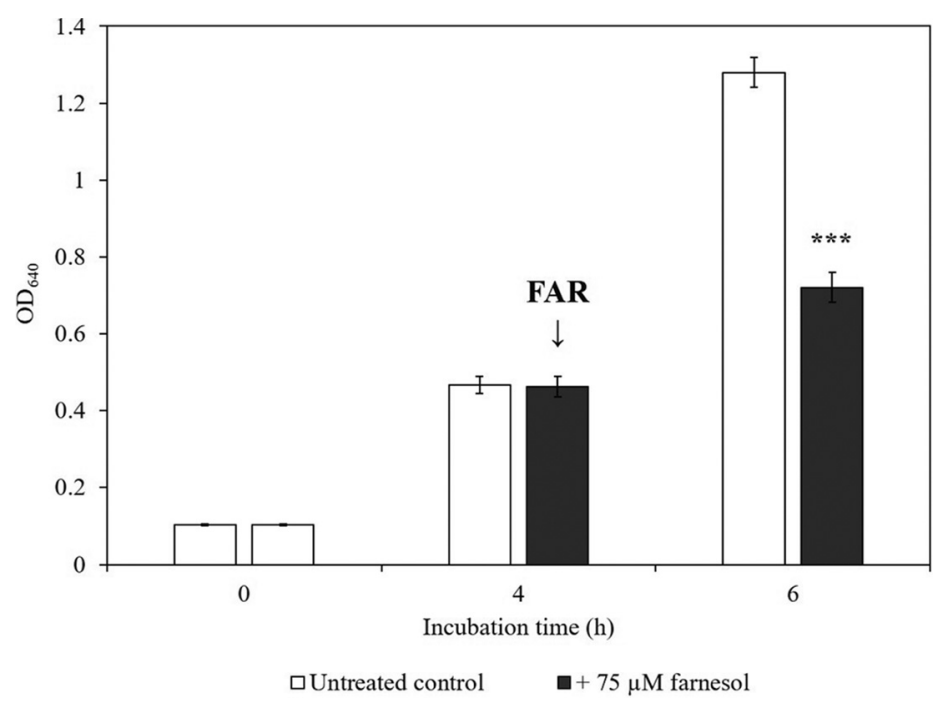

FIG 1 Farnesol exposure inhibits the growth of Candida auris. Changes in the growth of $C$. auris were monitored by measurement of the absorbance $\left(O D_{640}\right)$. Following a 4-h incubation time, farnesol (FAR) was added at a final concentration of $75 \mu \mathrm{M}$ to the YPD cultures. Data represent mean values \pm standard deviations (SD) (error bars) calculated from six independent experiments. The asterisks indicate a statistically significant difference between control and farnesol-treated cultures calculated by paired Student's $t$ test $\left({ }^{* *}, P<0.001\right)$.

Transcriptional profiling and RNA-Seq data validation. Principal-component analysis (PCA) and hierarchical clustering were performed to provide a visual representation of the transcriptomic similarities between samples treated with farnesol and the untreated controls (Fig. 3A and B). Samples from different conditions (with or without farnesol) clustered separately, whereas those from the same conditions clustered together, indicating a high level of correlation among samples as well as distinctive transcriptome profiles. Analyses of the RNA sequencing data clearly indicated that farnesol has a remarkable effect on $C$. auris gene transcription, leading to significant alterations in the transcriptome.

Comparison of the farnesol-exposed $C$. auris global gene transcription profile with that of unexposed cells revealed 1,766 differentially expressed genes. Among these genes, 447 were upregulated and 304 were downregulated in the farnesol-exposed samples compared to the untreated controls (Fig. 4 and 5; see also Tables S2 and S3 in the supplemental material).

Evaluation of farnesol-responsive genes. To identify larger patterns in differential gene transcription and to obtain an overall insight into the impact of farnesol, gene ontology (GO) terms were assigned to all of the genes in the C. auris genome; afterwards, we compared the terms for both the downregulated and upregulated genes to a background of all terms. We found 19 and 22 significant gene groups that were underrepresented and overrepresented in this analysis, respectively (Fig. 5 and Tables S2 and S3).

(i) Virulence-related genes. Virulence-related genes were significantly enriched within the farnesol-responsive downregulated gene group, according to Fisher's exact test (Table S3).

Most of these 11 putative genes are involved in biofilm maturation (RBT1, HWP1, $B C R 1, E F G 1, D S E 1, B R G 1$, UME6, ZAP1, and RLM1) and dispersion (NRG1 and UME6) (Fig. 4 and 5 and Table S3); also, five downregulated morphogenesis genes (EFG1, HWP1, HGC1, WAL1, and VRP1) are notable (Fig. 4 and Table S3). Downregulation of RBT1 and NRG1 under farnesol treatment was also supported by reverse transcriptasequantitative PCR (RT-qPCR) data (Fig. 6 and Table S4).

(ii) Oxidative stress-related genes. Genes belonging to antioxidative defenserelated GO terms were enriched in the farnesol-responsive upregulated gene group 
A

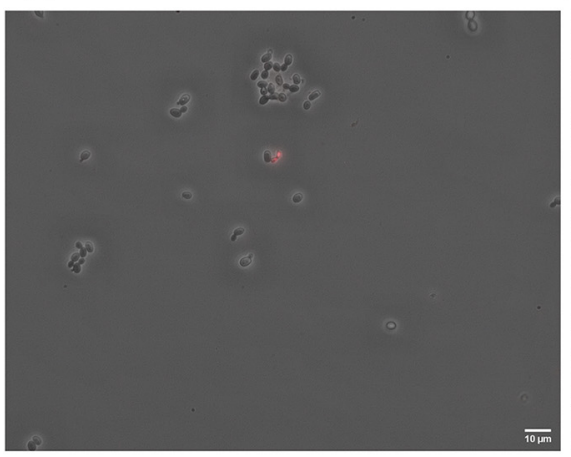

C

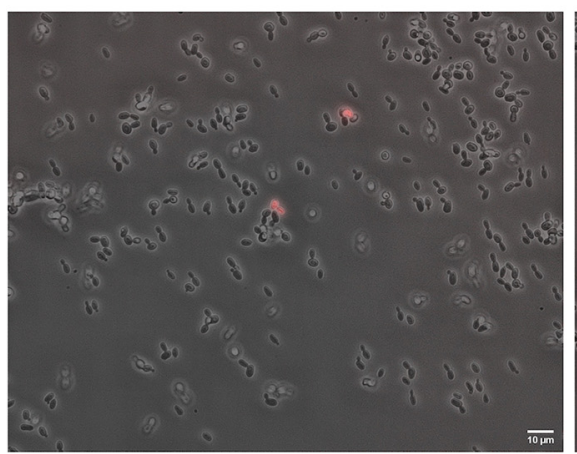

B

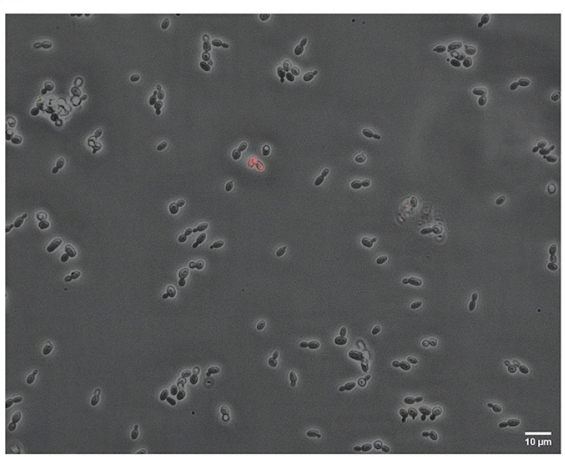

D

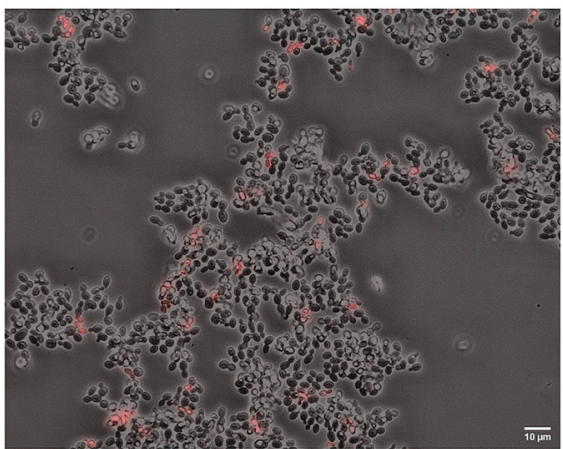

FIG 2 Phase-contrast and fluorescence microscopy. Phase-contrast and fluorescence microscopy images of untreated $C$. auris cells at 0,4 , and $6 \mathrm{~h}(\mathrm{~A}, \mathrm{~B}$, and $\mathrm{C}$, respectively) and cells treated with $75 \mu \mathrm{M}$ farnesol at $6 \mathrm{~h}$ (D). Propidium iodide fluorescent dye was used to stain the nonviable cells (red). Bars, $10 \mu \mathrm{m}$.

(Fig. 4 and 5 and Table S3). Altogether, eight genes were upregulated after farnesol treatment, namely, CCP1, SOD1, SOD2, SOD6, GPX1, DOT5, PRX1, and AHP1 (Fig. 4 and Table S3). In addition, farnesol exposure increased the transcription of HSP21, $Y P D 1$, and $H O G 1$, encoding small heat shock protein, phosphorelay protein, and mitogen-activated protein (MAP) kinase (Fig. 4 and Table S3). Upregulation of CAT1, coding for catalase in farnesol-treated cells, was also confirmed by RT-qPCR (Fig. 6 and Table S4).

(iii) Metabolic pathway-related genes. Selected genes involved in glucose catabolism and fatty acid metabolism were determined with the Candida Genome Database (http://www.candidagenome.org). Farnesol treatment downregulated PCK1 and FBP1, encoding key enzymes specific to gluconeogenesis, but not glycolysis and tricarboxylic acid cycle genes (Fig. 4 and Table S3). In addition, three genes related to the glyoxylate cycle (ACO2, ICL1, and MDH1-3) were significantly enriched in the upregulated gene set (Fig. 4 and Table S3).

Significant upregulation of five putative genes encoding fatty acid $\beta$-oxidation enzymes was observed (POX1, ECI1, FAT1, FAA21, and POT1) (Fig. 4 and 5 and Tables S2 and S3). In addition, farnesol exposure decreased the transcription of INO1, encoding inositol-1-phosphate synthase (Fig. 4 and Table S3).

Genes involved in iron homeostasis, including essential elements of reductive iron uptake (FRE10, FET31, SMF12, and FTR1), siderophore transport (SIT1), and hemoglobin use (RBT5), as well as manganese (SMF1, transporter), copper uptake (CRP1, transporter), and zinc metabolism (CSR1, transcription factor), were enriched in the downregulated gene set (Fig. 4 and 5 and Table S3).

The upregulation of POT1 (3-oxoacyl coenzyme A [CoA] thiolase) and the downregulation of INO1 and FTR1 were supported by RT-qPCR data (Fig. 6 and Table S4). 
A

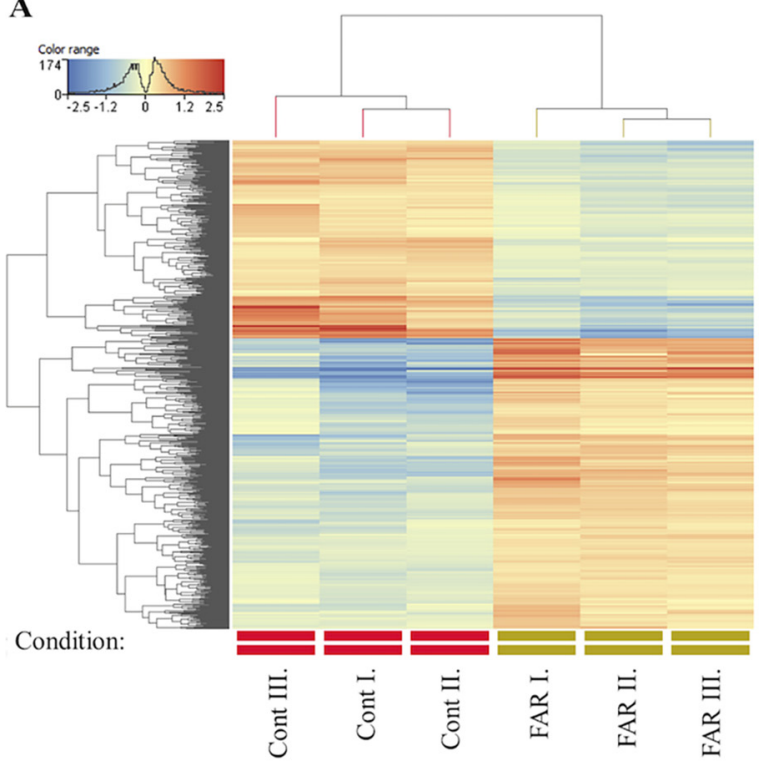

B

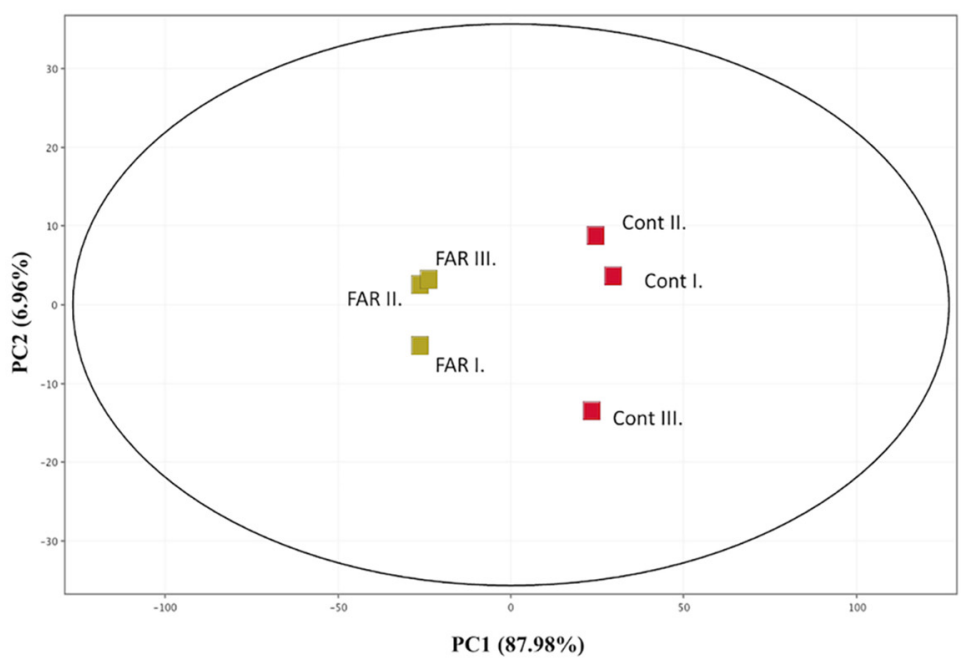

FIG 3 Cluster (A) and principal component (B) analysis of the transcriptome data. Symbols represent untreated control (Cont) and $75 \mu \mathrm{M}$ farnesol exposure (FAR) cultures. The distribution of transcriptome data obtained in three independent series of experiments (I, II, and III). Analyses were performed with the StrandNGS software using default settings.

(iv) Transmembrane transport-related genes. Farnesol treatment led to the increased transcription of numerous genes (60 genes altogether) involved in transmembrane transport, including 5 putative antifungal drug transporter genes (MDR1, CDR1, CDR4, HOL3, and YOR1), 4 putative carbohydrate transport genes (HGT2, HGT17, HGT19, and HXT5), 13 putative amino acid transport genes, as well as 4 putative phosphate and sulfate transporter genes (PHO84, PHO89, GIT1, and SUL2) (Fig. 4 and 5 and Table S3). Farnesol exposure also caused a significant increase in the transcription of CDR1 and MDR1 (ABC transporters) as well as HGT2 (glucose transmembrane transporter) of treated cells, according to the RT-qPCR results (Fig. 6 and Table S4).

Lipase: $L I P I 0$

Morphology: $E F G 1, H W P 1, H G C 1, W A L 1$, VRPI

Biofilm- Maturation: RBTl, $H W P I, B C R I$, EFGI, DSE1, BRGI, UME6, ZAPI, RLMI Biofilm- Dispersion: NRG1, UME6

Cell wall organization: $E R G 6, K R E 6, E N G 1$, CHT3, RLMI, PIRI, CBKI, C2 $10740 \mathrm{C}$

\begin{tabular}{l}
\hline Metabolism \\
Gluconeogenesis: MAEI, PCKI, FBPI \\
Inositol: INO1 \\
Iron metabolism: FRE10, FTRI, FET31, SITI, \\
SMFI2, RBT5 \\
RNA biosynthesis: TUPI, HAP42, SFU1, \\
DOT6, UME6, RLMI, BCRI, FLO8, RAD9, \\
MBPI, KAR4, STE20, CLN3, REGI, TPAI etc.
\end{tabular}

\begin{tabular}{l}
\hline Transport \\
Drug: $S N Q 2$ \\
Copper transport: $C R P 1$ \\
Zine transport: $C S R 1$ \\
Manganese transport: $S M F 1$ \\
\hline Signal transduction: $S S K 2, P B S 2, C M K 1$, \\
CBKI, CST20, FUN31, PRRI, KIS2, BUB1, \\
PTC8, SHA3 \\
\hline
\end{tabular}

Heat shock proteins: HSPI04, HSP78, HSP70, HSF $1, H S P 2 I$

Cell wall organization: $P H R I, X O G I, K E R Q$, YPS1, RBR2, SSP2, CHR11, FKS2, EXG2

Oxidative stress defence

Antioxidant activities: CATI, CCPI, SODI, SOD2, SOD6, GPX1, DOT5, AHP1, PRX1

\section{Metabolism}

Fatty acid oxidation: POXI, ECII, FATI FAA21, POT1

Glyoxylate cycle: $A C O 2, I C L 1, M D H 1-3$

\section{Transpor}

Drug: MDRI, CDR1, CDR4, HOL3, YORI Carbohydrate: HGT2, HGT17, HGT19, HXT5 Succinate/fumarate: $S F C l$

Amino acid: $U G A 4$, GAP2, DIP5, PUT4, AGP3, MUP3, PEX2, PEX5, PEX12, AVT4, OPT1, OPT2, SLY41

Nucleosid/nucleobase: FUR4, XUTI, DAL4, C4_00680W

Calcium: PMC1 Potassium: C3_01680C Heme: $F L C 2$

Ammonium: $M E P 1, M E P 2$ Phosphate: $P H O 84$ PHO89, GITI Sulfate: SUL2

Signal transduction: $H O G I, Y P D I, Z N C I$. PDK2, Cl 08780W, C5 00750C

FIG 4 Overview of transcriptional changes induced by farnesol in C. auris. Upregulated (red) and downregulated (blue) genes were defined as differentially expressed genes (corrected $P$ value of $<0.05$ ), with more than a 1.5 -fold increase or decrease in their transcription (farnesol treated versus untreated). On the sides of the volcano plot are representative genes upregulated or downregulated by farnesol treatment. The data set is available in Table $\mathrm{S} 3$ in the supplemental material. 
$\mathbf{A}$

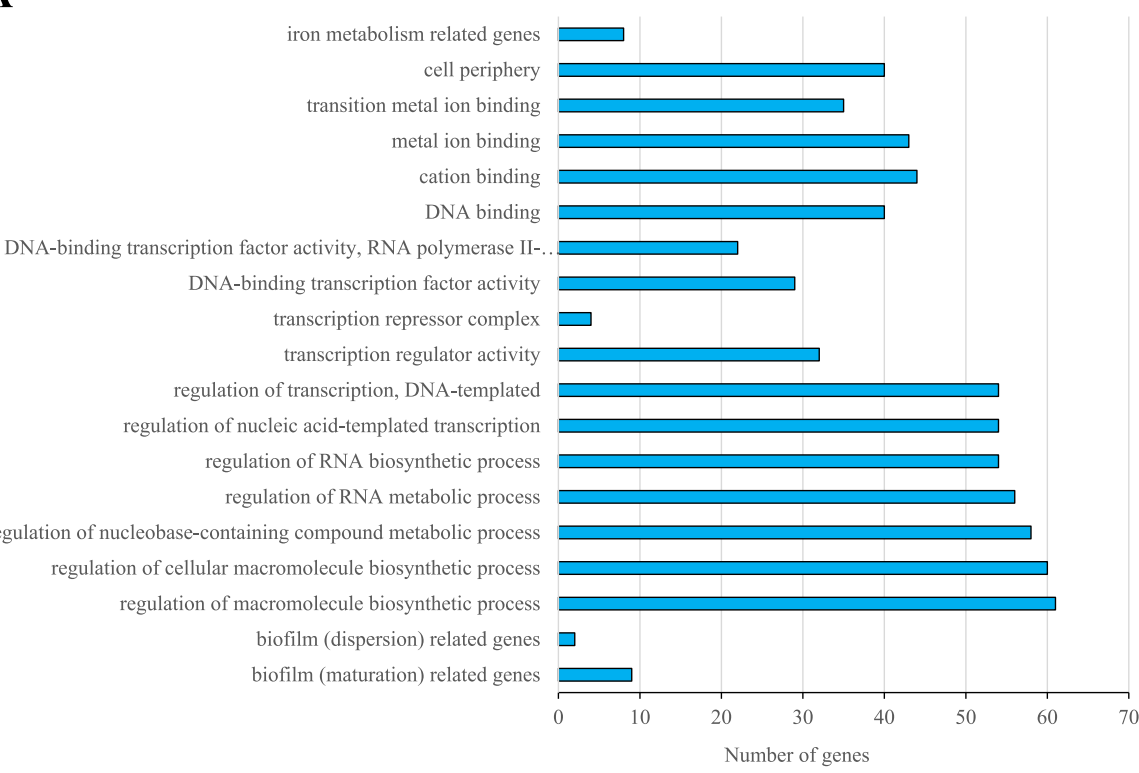

B

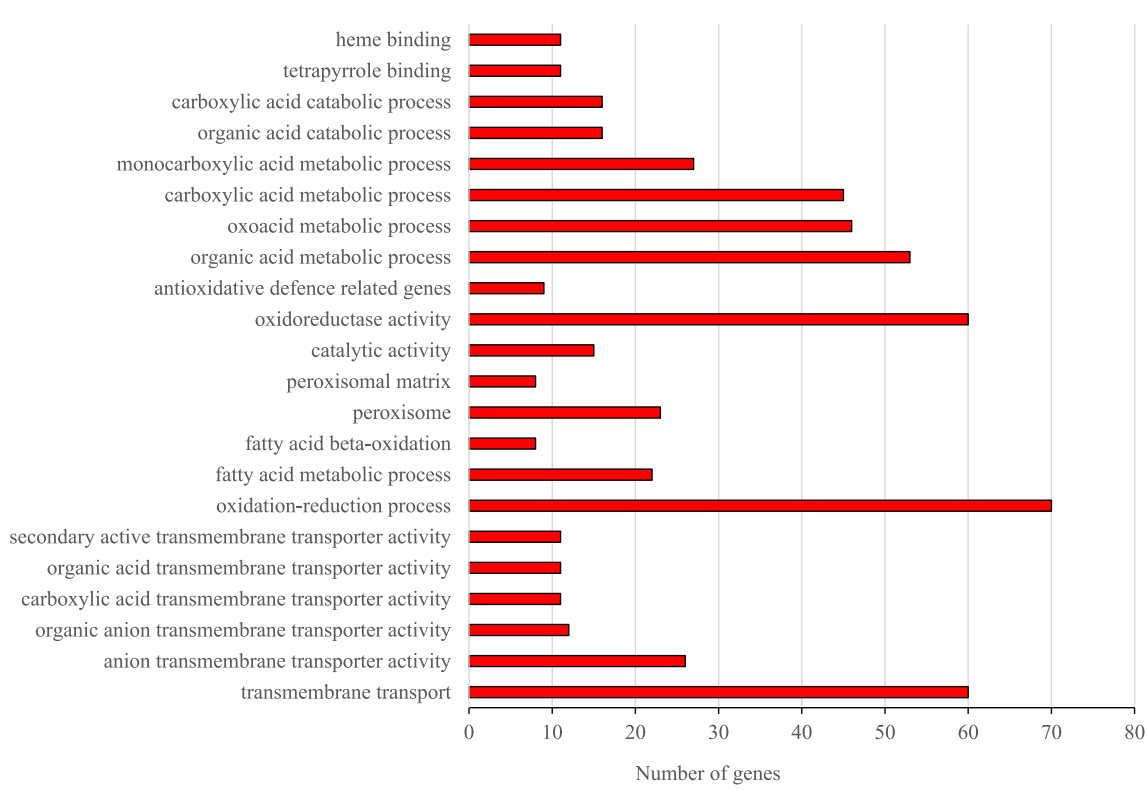

FIG 5 Summary of gene enrichment analyses and the number of genes affected by farnesol exposure of $C$. auris. Downregulated (blue) (A) and upregulated (red) (B) genes were defined as differentially expressed genes (corrected $P$ value of $<0.05$ ). The enrichment of these gene groups was identified with the Candida Genome Database Gene Ontology Term Finder (http://www.candidagenome.org/cgi-bin/GO/goTermFinder) or was tested by Fisher's exact test. The data sets for the gene groups are available in Tables S2 and S3 in the supplemental material.

Farnesol exposure significantly influences the metal contents of $C$. auris cells. Farnesol treatment caused a 24\%,73\%, and 69\% reduction in intracellular iron, manganese, and zinc content, respectively, compared to untreated control cells $(152.2 \pm 21.1 \mathrm{mg} / \mathrm{kg}$ versus $116.0 \pm 10.0 \mathrm{mg} / \mathrm{kg}, 67.9 \pm 5.1 \mathrm{mg} / \mathrm{kg}$ versus $18.6 \pm 1.8 \mathrm{mg} / \mathrm{kg}$, and $787.8 \pm 22.2 \mathrm{mg} / \mathrm{kg}$ versus $245.8 \pm 34.4 \mathrm{mg} / \mathrm{kg}$, for iron, manganese, and zinc, respectively) ( $P<0.05$ to 0.001$)$, whereas the level of intracellular copper showed a $302 \%$ increase $(274.6 \pm 15.7 \mathrm{mg} / \mathrm{kg}$ versus $828.8 \pm 106.4 \mathrm{mg} / \mathrm{kg})$, as shown in Table $1(P<0.001)$. 


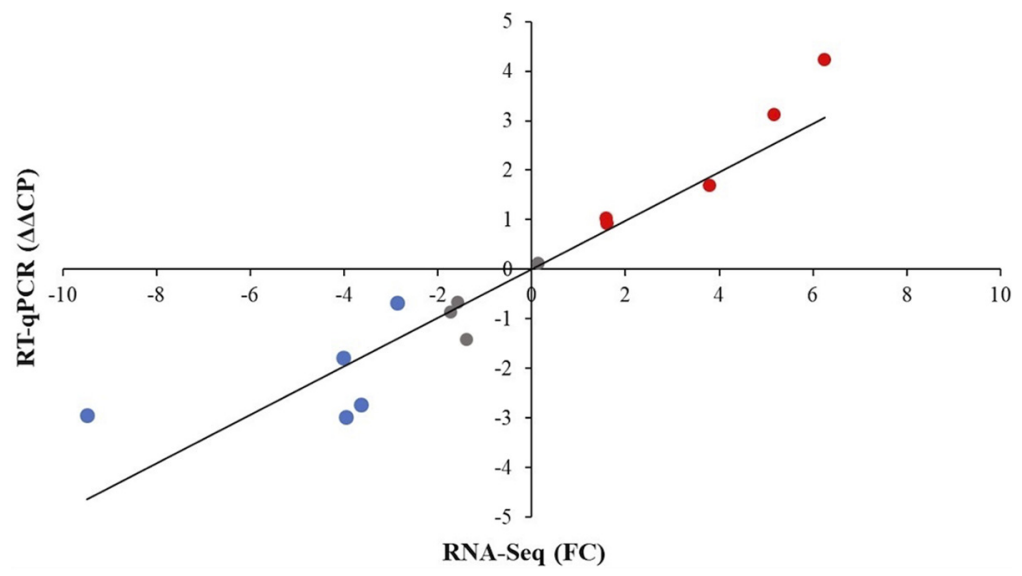

FIG 6 Correlation between RT-qPCR and transcriptome data. The expression patterns of genes related to biofilm formation (RBT5 and NRG1), oxidation-reduction (CAT1), membrane transport (MDR1, CDR1, HGT2, and FTR1), and metabolism (PFK1, PDC12, ADH1, INO1, POT1, and ERG1) were confirmed by the RT-qPCR assays. RNA-Seq data are presented as fold change (FC) values. Relative transcription levels were quantified as $\Delta \Delta C P=\Delta C_{\text {control }}-\Delta C P_{\text {treated }}$ where $\Delta \mathrm{CP}_{\text {treated }}=C_{\text {tested gene }}-C \mathrm{CP}_{\text {reference gene' }}$ measured from farnesol-treated cultures, and $\Delta C P_{\text {control }}=C P_{\text {tested gene }}-C_{\text {reference gene, }}$ measured from control cultures. CP values represent the qRT-PCR cycle numbers of crossing points. The ACT1 gene was used as a reference gene. $\Delta \Delta C P$ values significantly ( $P<0.05$ by Student's $t$ test; $n=3$ ) higher or lower than zero (up- or downregulated genes) are indicated in red and blue, respectively. Pearson's correlation coefficient between the RT-qPCR and RNA-Seq values was 0.87. The data set is available in Table S4.

\section{DISCUSSION}

Alternative treatments interfering with quorum sensing have recently become attractive therapeutic strategies, particularly against difficult-to-treat multidrug-resistant pathogens such as $C$. auris $(9,15,16)$. Previous studies have reported that fungal quorum-sensing molecules may have a remarkable antifungal effect and/or a potent adjuvant effect in combination with traditional antifungal agents (7, 10, 17-20). For example, Nagy et al. reported that supraphysiological farnesol exposure caused a significant reduction in the growth rate and metabolic activity of $C$. auris planktonic cells and biofilms, respectively (7). In addition, $75 \mu \mathrm{M}$ farnesol treatment significantly decreased the fungal kidney burden in an immunocompromised systemic mouse model (7). Total transcriptome analysis using RNA-Seq may be an important technique to fully understand the underlying mechanisms of the observed antifungal effect exerted by these molecules. In C. albicans, the transcription level of several genes has been shown to be affected by supraphysiological farnesol. Cao et al. (21) reported that farnesol exposure caused an increased expression of TUP1 (related to morphogenesis), FCR1 (drug resistance gene), FTR2 (iron transport gene), and CHT2 and CHT3 (chitinase genes). CSH1 (related to cell surface hydrophobicity) had a downregulation response in the presence of farnesol similar to HSP70, HSP90, and SSA2 (encoding heat shock proteins), PDR16 (drug resistance gene), and CRK1 and PDE2 (related to morphogenesis) $(8,21)$.

On the basis of previous studies, farnesol induces a dose-dependent production of reactive species in C. albicans, especially at supraphysiological concentrations $(22,23)$. Moreover, farnesol influences the transcription of CAT1, SOD1, and SOD2, which were

TABLE 1 Farnesol exposure significantly influences the metal contents of Candida auris cells

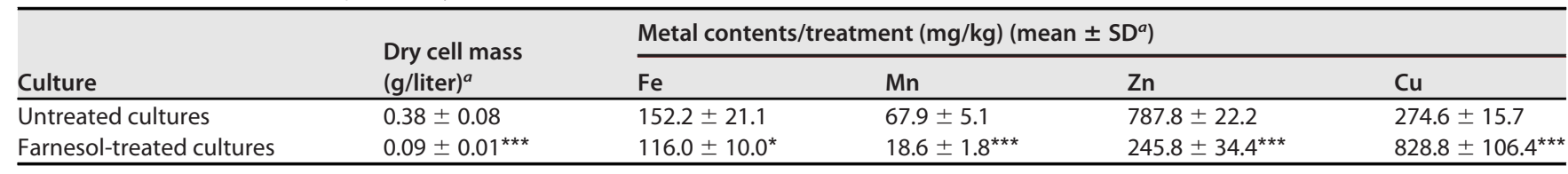

a Mean values \pm standard deviations (SD) calculated from three independent experiments are presented. The asterisks indicate significant differences calculated by two-way ANOVA comparing untreated control and farnesol-treated cultures as follows: ${ }^{*}, P<0.05 ;{ }^{* * *}, P<0.001$. 
linked to the oxidative stress response in C. albicans (8). These findings coincided with the C. auris-related physiological experiments published by Nagy et al. (7). In this study, several putative oxidative stress-responsive genes, namely, CAT1 (encoding catalase activity), GPX1 (encoding glutathione peroxidase), and SOD1, SOD2, and SOD6 (encoding superoxide dismutases), were upregulated following exposure to farnesol. It is noteworthy that farnesol exposure also upregulated HOG1 MAP kinase, which is a critical component of the fungal oxidative stress response, further supporting the farnesol-induced oxidative stress in C. auris (24). This fact is further confirmed by the elevated $2^{\prime}, 7^{\prime}$-dichlorofluorescein (DCF) and superoxide dismutase levels in farnesol-exposed cultures (7).

Recent transcriptomic data have demonstrated that farnesol treatment affected the transcription of iron homeostasis-related genes, as well as the iron, zinc, manganese, and copper contents of $C$. auris. The downregulation of iron uptake genes was associated with the significantly decreased iron content measured in farnesol-exposed cells. Similarly, the menadione sodium bisulfite-induced oxidative stress also affected the transcription of iron homeostasis-related genes and the iron content of $C$. albicans cells (25). It should be noted that this response related to iron decrease may be a part of a general defense mechanism against farnesol and menadione sodium bisulfite to minimize the damage caused by ferrous ions. According to previous studies, elevated free intracellular iron levels facilitate the formation of reactive oxygen species and mediate iron-dependent cell death in Saccharomyces cerevisiae $(25,26)$.

The downregulated transcription of CSR1, encoding a major transcription factor that stabilizes zinc homeostasis and provides cells with zinc-dependent protection against farnesol-induced oxidative stress (14), is related to the decreased intracellular zinc level observed. Zinc is an essential transition metal in oxidative stress defense because it is a structural component of superoxide dismutase, which is a key enzyme in the neutralization of superoxide radical anions $\left(\mathrm{O}_{2}{ }^{-}\right)(14)$.

In contrast to the majority of metals, manganese acts as an antioxidant element at high concentrations rather than a reactive oxygen species producer (14). However, farnesol inhibited the transcription of SMF1, which is responsible for maintaining the intracellular manganese levels for antioxidant actions (14). In addition, the transcription of PMR1 ( $P<0.05$, fold change $[F C]=1.2)$ was also inhibited, decreasing the virulence of fungal cells (27). This was associated with our previously published data, where daily farnesol treatment significantly decreased the virulence of $C$. auris (7).

The observed downregulation of the copper exclusion system (CRP1 and/or CCC2, encoding P-type ATPases) may be associated with the significantly increased copper contents and the remarkable growth inhibition in farnesol-treated cells. Copper regulates a variety of cellular processes in fungal pathogens. When it presents in excess, it is associated with the generation of reactive oxygen species via the Fenton reaction and destroys the iron-sulfur cluster reducing the viability of cells $(14,28-31)$. The elevated free copper levels in the farnesol-exposed cells may contribute to the increased redox imbalance quantified by DCF production (7), which was accompanied by increases in the specific activity of superoxide dismutase (7). Moreover, recent studies have shown that copper efflux pumps may be equally important in fungal defense strategies against phagocytes as for the virulence in C. albicans $(14,28-31)$.

Interestingly, farnesol exposure exerted a significant upregulation in several fatty acid $\beta$-oxidation-related genes (POX1, ECI1, FAT1, FAA21, and POT1). The elimination of unnecessary membrane lipids and the increased usage of fatty acids may provide a higher metabolic flux, needed for the maintenance of membrane fluidity (32). JabraRizk et al. (5) and Rossignol et al. (6) reported that farnesol influences the membrane permeability in non-albicans species such as Candida dubliniensis and Candida parapsilosis. The elevated fatty acid oxidation activity may explain the membrane-related farnesol effect, which may elucidate the previously observed antifungal effect (7). A further potential explanation of the antifungal effect can be found in the downregulation of ergosterol biosynthesis-related genes, which alter the membrane permeability and/ or fluidity (33). Dižová et al. reported that the presence of $200 \mu \mathrm{M}$ farnesol 
downregulated the ERG20,ERG11, and ERG9 genes in C. albicans (33). Based on these facts, exogenous farnesol has an effect on the synthesis of ergosterol.

In our study, the ERG6 gene was downregulated following farnesol exposure, which may enhance the passive diffusion of farnesol across the membrane; furthermore, the decreased Erg6 content may confirm the higher susceptibility of $C$. auris cells to oxidative stress $(34,35)$. Oliveira et al. (34) showed that the ERG6 mutant Cryptococcus neoformans displays impaired thermotolerance and increased susceptibility to oxidative stress as well as to different antifungal drugs, explaining, for instance, the previously reported synergizing effect with azoles $(7,34)$. Furthermore, the ERG6 mutant $C$. neoformans was totally avirulent in an invertebrate model, which may also explain the reduced virulence of $C$. auris after daily farnesol treatment $(7,34)$. Beside ERG6, INO1 was also downregulated following farnesol treatment. This gene encodes the inositol1-phosphate synthase, a key enzyme in the synthesis of inositol for phosphotidylinositol synthesis. The downregulation of this gene may further explain the synergizing effect of farnesol with azoles against C. auris (7), because INO1 is significantly upregulated in drug-resistant Candida isolates (21).

With respect to the transport efflux pump-related genes, farnesol exposure caused a significant increase in the transcription of CDR1, CDR4, MDR1, HOL3, and YOR1, whereas the transcription level of SNQ2 was decreased. Previous studies have revealed that these transporters mediate drug resistance for $C$. auris $(36,37)$. Srivastava and Ahmad found that CDR1, CDR2, MDR1, MDR2, and SNQ2 are significantly downregulated in the presence of farnesol (38). Notably, there was a 1,000-fold difference between the farnesol dosages exerting the upregulating effect (125 mM) compared to the concentration used in our study $(75 \mu \mathrm{M})$. Nevertheless, our data support the hypothesis that farnesol, at lower concentrations, may be a potential substrate for the upregulated transport proteins in order to protect the cells themselves from the oxidative stress induced by farnesol.

This is the first study analyzing the global changes in gene transcription in C. auris following farnesol exposure, providing important insights into the mechanism of antifungal action of farnesol and the response of $C$. auris, facilitating a better understanding of farnesol-related antifungal activity. In summary, farnesol exposure enhanced the oxidative stress response and upregulated drug efflux pumps, while reducing zinc and manganese intracellular content as well as iron metabolism. Moreover, cellular metabolism was modulated toward $\beta$-oxidation. These findings reveal the mechanisms underlying the antifungal effect and suggest that farnesol may represent a potent therapeutic option against this multiresistant fungal superbug.

\section{MATERIALS AND METHODS}

Fungal strain, media, and culture conditions. The C. auris isolate 12 (NCPF 8973), belonging to the South Asian/Indian lineage, was obtained from the National Mycology Reference Laboratory (United Kingdom) (39). For the tested C. auris isolate, the MICs were $0.125 \mathrm{mg} /$ liter, $1 \mathrm{mg} /$ liter, $0.125 \mathrm{mg} /$ liter, $4 \mathrm{mg} /$ liter, and $0.25 \mathrm{mg} /$ liter for anidulafungin, caspofungin, micafungin, fluconazole, and amphotericin $B$, respectively.

The test strain was maintained and cultured on yeast extract-peptone-dextrose (YPD) agar (1\% yeast extract [Alfa Aesar, USA], 2\% mycological peptone [Oxoid, UK], 2\% dextrose [VWR International LIc., Hungary], with or without 2\% agar [VWR International LIc., Hungary] [pH 5.6]) as described previously (40).

To study the effect of farnesol on short-term transcriptional response, $C$. auris precultures were grown in $5 \mathrm{ml} \mathrm{YPD}$ medium at $30^{\circ} \mathrm{C}$ at a shaking frequency of $2.3 \mathrm{~Hz}$ for $18 \mathrm{~h}$. Subsequently, the inoculum was diluted to an optical density of 0.1 at $\lambda=640 \mathrm{~nm}\left(\mathrm{OD}_{640}\right)$ with YPD (at $0-\mathrm{h}$ incubation time as defined in growth assays), and the cultures were further grown at $37^{\circ} \mathrm{C}$ and $2.3-\mathrm{Hz}$ shaking frequency. At 4-h incubation time, the cultures were supplemented with $75 \mu \mathrm{M}$ farnesol, and microbial growth was monitored by measuring changes in $\mathrm{OD}_{640}$ and $\mathrm{CFU}(7,41)$. Farnesol (Merck, Budapest, Hungary) was obtained as a $3 \mathrm{M}$ stock solution that was diluted to a $30 \mathrm{mM}$ working stock solution in $100 \%$ methanol. The working concentrations were prepared in YPD. Farnesol-free control flasks contained 1\% (vol/vol) methanol. Growth was evaluated in six independent experiments and is presented as the mean \pm standard deviation (SD). Statistical comparison of growth-related data was performed by paired Student's $t$ test. The differences between values for treated and control cells were considered significant at a $P$ value of $<0.05$. 
Microscopy. Farnesol-induced morphological and viability changes were examined at $75 \mu \mathrm{M}$ after 0 , 4 , and $6 \mathrm{~h}$ of incubation at $37^{\circ} \mathrm{C}$. Afterwards, $999 \mu \mathrm{l}$ of the culture was stained with $1 \mu \mathrm{l}$ of $20 \mathrm{mM}$ propidium iodide (ThermoFisher, Waltham, MA, USA). Fluorescently stained cells were incubated further at $37^{\circ} \mathrm{C}$ for $30 \mathrm{~min}$; then, $10 \mu \mathrm{l}$ of medium was mounted on a slide and examined using a Zeiss Axioskop 2 mot microscope coupled with a Zeiss Axiocam HRc camera using the phase-contrast and fluorescent technique to assess cell morphology and the ratio of nonviable cells, respectively. Further picture analysis and calculation of the percentage of the dead cells were performed using ImageJ software (version 2.1.0/1.53c) (Fiji, ImageJ; Wayne Rasband, National Institutes of Health) (42).

RNA isolation and sequencing. Total RNA was extracted from untreated control cells and $75 \mu \mathrm{M}$ farnesol-treated cultures in three biological replicates. Briefly, fungal cells were collected at $2 \mathrm{~h}$ following farnesol exposure by centrifugation ( $5 \mathrm{~min}$ at a relative centrifugal force [RCF] of $4,000 \times g$ at $4^{\circ} \mathrm{C}$ ). The cells were washed three times with phosphate-buffered saline (PBS) and stored at $-70^{\circ} \mathrm{C}$ until use. Total RNA samples were prepared from freeze-dried cells (CHRIST Alpha 1-2 LDplus lyophilizer, Osterode, Germany) derived from untreated and farnesol-treated cultures using TRIzol (Invitrogen, Austria) reagent by the method of Chomczynski et al. (43). To determine the final RNA concentration and quality, samples were analyzed on an Agilent BioAnalyzer using the Eukaryotic Total RNA Nano kit (Agilent Technologies, Inc., Santa Clara, CA, USA) according to the manufacturer's protocol. Samples with RNA integrity number (RIN) values of $>7$ were accepted for the library preparation process. Three independent cultures were used for RNA-Seq experiments and RT-qPCR tests.

To obtain global transcriptome data, high-throughput mRNA sequencing was performed. The RNA-Seq libraries were prepared from total RNA using the NEBNext Ultra II RNA sample preparation kit (NEB, USA) according to the manufacturer's protocol. The single-read 75-bp-long sequencing reads were generated on an Illumina NextSeq500 instrument. Approximately 18 to 22 million reads per samples were generated. The library preparations and the sequencing run were performed by the Genomic Medicine and Bioinformatics Core Facility of the Department of Biochemistry and Molecular Biology, Faculty of Medicine, University of Debrecen, Hungary. Raw reads were aligned to the reference genome (genome, https://fungi.ensembl.org/ _candida_auris_gca_002759435/Info/Index; features, http://www.candidagenome.org/download/gff/C_auris _B8441/archive/C_auris_B8441_version_s01-m01-r11_features_with_chromosome_sequences.gff.gz), and aligned reads varied between 90 and $95 \%$ in each sample. The DESeq algorithm (StrandNGS software) was used to obtain normalized gene transcription values. Gene transcription differences between farnesolexposed and control groups were compared by a moderated $t$ test; the Benjamini-Hochberg false discovery rate was used for multiple-testing correction, and a corrected $P$ value of $<0.05$ was considered significant (differentially expressed genes). Up- and downregulated genes were defined as differentially expressed genes with $>1.5$-fold change ( $F C$, upregulated genes) or less than $-1.5-F C$ (downregulated genes) values. The FC ratios were calculated from the normalized gene transcription values.

Reverse transcriptase-quantitative PCR assays. Changes in the transcription of selected oxidative stress response, membrane transport, virulence, and primary metabolism genes were validated by reverse transcriptase-quantitative PCR (RT-qPCR) (41). The RT-qPCRs with Luna Universal one-step RTqPCR kit (NEB, USA) were performed according to the protocol of the manufacturer, using $500 \mathrm{ng}$ of DNase (Sigma, Budapest, Hungary)-treated total RNA per reaction. Oligonucleotide primers (see Table S1 in the supplemental material) were designed with the software packages Oligo Explorer (version 1.1.) and Oligo Analyzer (version 1.0.2). Three parallel measurements were performed with each sample in a LightCycler 96 real-time PCR instrument (Roche, Switzerland). Relative transcription levels ( $\Delta \Delta C P$ value) were calculated as $\Delta \mathrm{CP}_{\text {control }}-\Delta \mathrm{CP}_{\text {treated }}$, where $\Delta \mathrm{CP}_{\text {control }}=\mathrm{CP}_{\text {tested gene, control }}-\mathrm{CP}_{\text {reference gene, control }}$ for untreated control, and $\Delta C P_{\text {treated }}=C P_{\text {tested gene, treated }}-C P_{\text {reference gene, treated }}$ for farnesol-exposed cultures (13). The CP values represent the RT-qPCR cycle numbers of crossing points. The reference gene used was ACT1 (B9J08_000486). The $\triangle \triangle C P$ values are expressed as mean \pm SD calculated from three independent measurements, and $\Delta \Delta C P$ values significantly $(P<0.05)$ higher or lower than zero were determined using the Student's $t$ test.

Functional enrichment analysis. Gene set enrichment analyses on the upregulated and downregulated gene sets were performed with Candida Genome Database Gene Ontology Term Finder (http:// www.candidagenome.org/cgi-bin/GO/goTermFinder), using function, process, and component gene ontology (GO) terms. Only hits with a $P$ value of $<0.05$ were considered in the evaluation process (Table S2).

Besides GO terms, groups of functionally related genes were also generated by extracting data from the Candida Genome Database (http://www.candidagenome.org) unless otherwise indicated. The enrichment of $C$. auris genes from these gene groups in the upregulated and downregulated gene sets was tested with Fisher's exact test $(P<0.05)$. The following gene groups were created.

(i) Virulence-related genes. Genes involved in the genetic control of $C$. albicans virulence were collected by the methods of Mayer et al. (44), Höfs et al. (45), and Araújo et al. (46).

(ii) Metabolic pathway-related genes. This group contains all genes related to the carbohydrate, ergosterol, and fatty acid biochemical pathways according to the pathway databases (http://pathway .candidagenome.org/).

(iii) Antioxidant enzyme genes. This group includes genes encoding functionally verified and/or putative antioxidant enzymes according to catalases (GOID:4096), SODs (GOID:4784), glutaredoxins (GOID:6749), thioredoxins (GOIDs:8379 and 51920), and peroxidases (GOID:4601) GO terms.

(iv) Iron metabolism-related genes. Genes involved in iron acquisition by $C$. albicans were collected by the method of Fourie et al. (47).

(v) Zinc, manganese, and copper homeostasis genes. Genes involved in zinc and copper acquisition were collected by the method of Gerwien et al. (14). 
The complete gene lists of the above-mentioned gene groups are available in Table S3.

Assays of iron, manganese, zinc, and copper contents of Candida auris cells. C. auris precultures were grown, and farnesol exposure was performed as described above. Yeast cells were collected by centrifugation ( $5 \mathrm{~min}, 4,000 \times \mathrm{g}, 4^{\circ} \mathrm{C}$ ) after $2 \mathrm{~h}$ of incubation following farnesol exposure. Changes in fungal dry cell mass (DCM) were determined after freeze-drying (25). The metal contents of the dry biomass were measured by inductively coupled plasma optical emission spectrometry (ICP-OES; 5110 Agilent Technologies, Santa Clara, CA, USA) following atmospheric wet digestion in $3 \mathrm{ml}$ of $65 \%$ (mass percent $[\mathrm{M} / \mathrm{M}]) \mathrm{HNO}_{3}$ and $1 \mathrm{ml}$ of $30 \%(\mathrm{M} / \mathrm{M}) \mathrm{H}_{2} \mathrm{O}_{2}$ in glass beakers. The metal contents of the samples were calculated and expressed in DCM units (in milligrams per kilogram) by the method of Jakab et al. (25). The metal contents of the biomasses were determined in triplicate, and mean \pm SD values were calculated. Statistical significance of changes was determined by two-way analysis of variance (ANOVA). Significance was defined as a $P$ value of $<0.05$.

Availability of data. The RNA sequencing data discussed have been deposited in NCBI's Gene Expression Omnibus (48) (GEO; http://www.ncbi.nlm.nih.gov/geo/) and are accessible through GEO Series accession number GSE180093.

\section{SUPPLEMENTAL MATERIAL}

Supplemental material is available online only.

TABLE S1, DOCX file, $0.01 \mathrm{MB}$.

TABLE S2, XLSX file, $0.02 \mathrm{MB}$.

TABLE S3, XLSX file, $0.1 \mathrm{MB}$.

TABLE S4, XLSX file, $0.01 \mathrm{MB}$.

\section{ACKNOWLEDGMENTS}

This project was supported by the EFOP-3.6.3-VEKOP-16-2017-00009 program. This research was funded by the European Union and the European Social (EFOP-3.6.1-162016-00022) by the Thematic Excellence Programme (TKP2020-IKA-04) of the Ministry for Innovation and Technology in Hungary. This research was funded by the Hungarian National Research, Development and Innovation Office (NKFIH FK138462) (R. Kovács). R. Kovács was supported by OTKA Bridging Fund and FEMS Research and Training Grant (FEMS-GO-2019-502). R. Kovács was supported by the János Bolyai Research Scholarship of the Hungarian Academy of Sciences. F. Nagy was supported by the ÚNKP-19-3 and ÚNKP-20-3 New National Excellence Program of the Ministry for Innovation and Technology.

L. Majoros received conference travel grants from MSD, Astellas, and Pfizer. All other authors declare no conflicts of interest.

\section{REFERENCES}

1. Chowdhary A, Tarai B, Singh A, Sharma A. 2020. Multidrug-resistant Candida auris infections in critically ill coronavirus disease patients, India, April-July 2020. Emerg Infect Dis 26:2694-2696. https://doi.org/10.3201/ eid2611.203504.

2. Magnasco L, Mikulska M, Giacobbe DR, Taramasso L, Vena A, Dentone C, Dettori S, Tutino S, Labate L, Di Pilato V, Crea F, Coppo E, Codda G, Robba C, Ball L, Patroniti N, Marchese A, Pelosi P, Bassetti M. 2021. Spread of carbapenem-resistant Gram-negatives and Candida auris during the COVID-19 pandemic in critically ill patients: one step back in antimicrobial stewardship? Microorganisms 9:95. https://doi.org/10.3390/microorganisms9010095.

3. Mulet Bayona JV, Tormo Palop N, Salvador García C, Fuster Escrivá B, Chanzá Aviñó M, Ortega García P, Gimeno Cardona C. 2021. Impact of the SARS-CoV-2 pandemic in candidaemia, invasive aspergillosis and antifungal consumption in a tertiary hospital. J Fungi 7:440. https://doi.org/10 $.3390 /$ jof7060440.

4. Hornby JM, Jensen EC, Lisec AD, Tasto JJ, Jahnke B, Shoemaker R, Dussault $P$, Nickerson KW. 2001. Quorum sensing in the dimorphic fungus Candida albicans is mediated by farnesol. Appl Environ Microbiol 67: 2982-2992. https://doi.org/10.1128/AEM.67.7.2982-2992.2001.

5. Jabra-Rizk MA, Shirtliff M, James C, Meiller T. 2006. Effect of farnesol on Candida dubliniensis biofilm formation and fluconazole resistance. FEMS Yeast Res 6:1063-1073. https://doi.org/10.1111/j.1567-1364.2006.00121.x.

6. Rossignol T, Logue ME, Reynolds K, Grenon M, Lowndes NF, Butler G. 2007. Transcriptional response of Candida parapsilosis following exposure to farnesol. Antimicrob Agents Chemother 51:2304-2312. https://doi.org/ 10.1128/AAC.01438-06.
7. Nagy F, Vitális E, Jakab Á, Borman AM, Forgács L, Tóth Z, Majoros L, Kovács R. 2020. In vitro and in vivo effect of exogenous farnesol exposure against Candida auris. Front Microbiol 11:957. https://doi.org/10.3389/ fmicb.2020.00957.

8. Rodrigues CF, Černáková L. 2020. Farnesol and tyrosol: secondary metabolites with a crucial quorum-sensing role in Candida biofilm development. Genes 11:444. https://doi.org/10.3390/genes11040444.

9. Kovács R, Majoros L. 2020. Fungal quorum-sensing molecules: a review of their antifungal effect against Candida biofilms. J Fungi 6:99. https://doi .org/10.3390/jof6030099.

10. Nagy F, Tóth Z, Daróczi L, Székely A, Borman AM, Majoros L, Kovács R. 2020. Farnesol increases the activity of echinocandins against Candida auris biofilms. Med Mycol 58:404-407. https://doi.org/10.1093/mmy/myz057.

11. Simm C, May RC. 2019. Zinc and iron homeostasis: target-based drug screening as new route for antifungal drug development. Front Cell Infect Microbiol 9:181. https://doi.org/10.3389/fcimb.2019.00181.

12. Vila T, Kong EF, Ibrahim A, Piepenbrink K, Shetty AC, McCracken C, Bruno V, Jabra-Rizk MA. 2019. Candida albicans quorum-sensing molecule farnesol modulates staphyloxanthin production and activates the thiol-based oxidative-stress response in Staphylococcus aureus. Virulence 10:625-642. https://doi.org/10.1080/21505594.2019.1635418.

13. Liu Z, Rossi JM, Myers LC. 2018. Candida albicans Zn cluster transcription factors Tac1 and Znc1 are activated by farnesol to upregulate a transcriptional program including the multidrug efflux pump CDR1. Antimicrob Agents Chemother 62:e00968-18. https://doi.org/10.1128/AAC.00968-18. 
14. Gerwien F, Skrahina V, Kasper L, Hube B, Brunke S. 2018. Metals in fungal virulence. FEMS Microbiol Rev 42:fux050. https://doi.org/10.1093/femsre/ fux050.

15. Grainha TRR, Jorge PADS, Pérez-Pérez M, Pérez Rodríguez G, Pereira MOBO, Lourenço AMG. 2018. Exploring anti-quorum sensing and anti-virulence based strategies to fight Candida albicans infections: an in silico approach. FEMS Yeast Res https://doi.org/10.1093/femsyr/foy022.

16. Fong J, Zhang C, Yang R, Boo ZZ, Tan SK, Nielsen TE, Givskov M, Liu XW, Bin W, Su H, Yang L. 2018. Combination therapy strategy of quorum quenching enzyme and quorum sensing inhibitor in suppressing multiple quorum sensing pathways of $P$. aeruginosa. Sci Rep 8:1155. https://doi .org/10.1038/s41598-018-19504-w.

17. Kovács R, Bozó A, Gesztelyi R, Domán M, Kardos G, Nagy F, Tóth Z, Majoros L. 2016. Effect of caspofungin and micafungin in combination with farnesol against Candida parapsilosis biofilms. Int J Antimicrob Agents 47:304-310. https://doi.org/10.1016/j.ijantimicag.2016.01.007.

18. Bozó A, Domán M, Majoros L, Kardos G, Varga I, Kovács R. 2016. The in vitro and in vivo efficacy of fluconazole in combination with farnesol against Candida albicans isolates using a murine vulvovaginitis model. J Microbiol 54:753-760. https://doi.org/10.1007/s12275-016-6298-y.

19. Katragkou A, McCarthy M, Alexander EL, Antachopoulos C, Meletiadis J, Jabra-Rizk MA, Petraitis V, Roilides E, Walsh TJ. 2015. In vitro interactions between farnesol and fluconazole, amphotericin B or micafungin against Candida albicans biofilms. J Antimicrob Chemother 70:470-478. https:// doi.org/10.1093/jac/dku374.

20. Fernandes Costa A, Evangelista Araujo D, Santos Cabral M, Teles Brito I, Borges de Menezes Leite L, Pereira M, Correa Amaral A. 2019. Development, characterization, and in vitro-in vivo evaluation of polymeric nanoparticles containing miconazole and farnesol for treatment of vulvovaginal candidiasis. Med Mycol 57:52-62. https://doi.org/10.1093/mmy/ myx155.

21. Cao YY, Cao YB, Xu Z, Ying K, Li Y, Xie Y, Zhu ZY, Chen WS, Jiang YY. 2005. cDNA microarray analysis of differential gene expression in Candida albicans biofilm exposed to farnesol. Antimicrob Agents Chemother 49: 584-589. https://doi.org/10.1128/AAC.49.2.584-589.2005.

22. Davis-Hanna A, Piispanen AE, Stateva LI, Hogan DA. 2008. Farnesol and dodecanol effects on the Candida albicans Ras1-cAMP signalling pathway and the regulation of morphogenesis. Mol Microbiol 67:47-62. https://doi .org/10.1111/j.1365-2958.2007.06013.x.

23. Deveau A, Piispanen AE, Jackson AA, Hogan DA. 2010. Farnesol induces hydrogen peroxide resistance in Candida albicans yeast by inhibiting the Ras-cyclic AMP signaling pathway. Eukaryot Cell 9:569-577. https://doi .org/10.1128/EC.00321-09.

24. Dantas AS, Day A, Ikeh M, Kos I, Achan B, Quinn J. 2015. Oxidative stress responses in the human fungal pathogen, Candida albicans. Biomolecules 5:142-165. https://doi.org/10.3390/biom5010142.

25. Jakab Á, Emri T, Csillag K, Szabó A, Nagy F, Baranyai E, Sajtos Z, Géczi D, Antal K, Kovács R, Szabó K, Dombrádi V, Pócsi I. 2021. The negative effect of protein phosphatase $\mathrm{Z} 1$ deletion on the oxidative stress tolerance of Candida albicans is synergistic with betamethasone exposure. J Fungi 7: 540. https://doi.org/10.3390/jof7070540.

26. Dixon SJ, Stockwell BR. 2014. The role of iron and reactive oxygen species in cell death. Nat Chem Biol 10:9-17. https://doi.org/10.1038/nchembio.1416.

27. Bates S, MacCallum DM, Bertram G, Munro CA, Hughes HB, Buurman ET, Brown AJ, Odds FC, Gow NA. 2005. Candida albicans Pmr1p, a secretory pathway P-type Ca2+/Mn2+-ATPase, is required for glycosylation and virulence. J Biol Chem 280:23408-23415. https://doi.org/10.1074/jbc .M502162200.

28. Weissman Z, Berdicevsky I, Cavari BZ, Kornitzer D. 2000. The high copper tolerance of Candida albicans is mediated by a P-type ATPase. Proc Natl Acad Sci U S A 97:3520-3525. https://doi.org/10.1073/pnas.97.7.3520.

29. Ding C, Festa RA, Sun TS, Wang ZY. 2014. Iron and copper as virulence modulators in human fungal pathogens. Mol Microbiol 93:10-23. https:// doi.org/10.1111/mmi.12653.

30. Mackie J, Szabo EK, Urgast DS, Ballou ER, Childers DS, MacCallum DM, Feldmann J, Brown AJ. 2016. Host-imposed copper poisoning impacts fungal micronutrient acquisition during systemic Candida albicans infections. PLoS One 11:e0158683. https://doi.org/10.1371/journal.pone.0158683.
31. Wiemann P, Perevitsky A, Lim FY, Shadkchan $Y$, Knox BP, Landero Figueora JA, Choera T, Niu M, Steinberger AJ, Wüthrich M, Idol RA, Klein BS, Dinauer MC, Huttenlocher A, Osherov N, Keller NP. 2017. Aspergillus fumigatus copper export machinery and reactive oxygen intermediate defense counter host copper-mediated oxidative antimicrobial offense. Cell Rep 19:1008-1021. https://doi.org/10.1016/j.celrep.2017.04.019.

32. Fradin C, Kretschmar M, Nichterlein T, Gaillardin C, d'Enfert C, Hube B. 2003. Stage-specific gene expression of Candida albicans in human blood. Mol Microbiol 47:1523-1543. https://doi.org/10.1046/j.1365-2958.2003 .03396.x.

33. Dižová S, Černáková L, Bujdáková H. 2018. The impact of farnesol in combination with fluconazole on Candida albicans biofilm: regulation of ERG20, ERG9, and ERG11 genes. Folia Microbiol (Praha) 63:363-371. https://doi.org/10.1007/s12223-017-0574-z.

34. Oliveira FFM, Paes HC, Peconick LDF, Fonseca FL, Marina CLF, Bocca AL, Homem-de-Mello M, Rodrigues ML, Albuquerque P, Nicola AM, Alspaugh JA, Felipe MSS, Fernandes L. 2020. Erg6 affects membrane composition and virulence of the human fungal pathogen Cryptococcus neoformans. Fungal Genet Biol 140:103368. https://doi.org/10.1016/j.fgb.2020.103368.

35. Jensen-Pergakes KL, Kennedy MA, Lees ND, Barbuch R, Koegel C, Bard M. 1998. Sequencing, disruption, and characterization of the Candida albicans sterol methyltransferase (ERG6) gene: drug susceptibility studies in erg6 mutants. Antimicrob Agents Chemother 42:1160-1167. https://doi .org/10.1128/AAC.42.5.1160.

36. Muñoz JF, Gade L, Chow NA, Loparev VN, Juieng P, Berkow EL, Farrer RA, Litvintseva AP, Cuomo CA. 2018. Genomic insights into multidrug-resistance, mating and virulence in Candida auris and related emerging species. Nat Commun 9:5346. https://doi.org/10.1038/s41467-018-07779-6.

37. Kean R, Delaney C, Sherry L, Borman A, Johnson EM, Richardson MD, Rautemaa-Richardson R, Williams C, Ramage G. 2018. Transcriptome assembly and profiling of Candida auris reveals novel insights into biofilm-mediated resistance. mSphere 3:e00334-18. https://doi.org/10.1128/mSphere.00334-18.

38. Srivastava V, Ahmad A. 2020. Abrogation of pathogenic attributes in drug resistant Candida auris strains by farnesol. PLoS One 15:e0233102. https://doi.org/10.1371/journal.pone.0233102.

39. Borman AM, Szekely A, Johnson EM. 2017. Isolates of the emerging pathogen Candida auris present in the UK have several geographic origins. Med Mycol 55:563-567. https://doi.org/10.1093/mmy/myw147.

40. Szabó K, Jakab Á, Póliska S, Petrényi K, Kovács K, Issa LHB, Emri T, Pócsi I, Dombrádi V. 2019. Deletion of the fungus specific protein phosphatase $\mathrm{Z1}$ exaggerates the oxidative stress response in Candida albicans. BMC Genomics 20:873. https://doi.org/10.1186/s12864-019-6252-6.

41. Jakab Á, Tóth Z, Nagy F, Nemes D, Bácskay I, Kardos G, Emri T, Pócsi I, Majoros L, Kovács R. 2019. Physiological and transcriptional responses of Candida parapsilosis to exogenous tyrosol. Appl Environ Microbiol 85: e01388-19. https://doi.org/10.1128/AEM.01388-19.

42. Kovács R, Nagy F, Tóth Z, Forgács L, Tóth L, Váradi G, Tóth GK, Vadászi K, Borman AM, Majoros L, Galgóczy L. 2021. The Neosartorya fischeri antifungal protein 2 (NFAP2): a new potential weapon against multidrug-resistant Candida auris biofilms. Int J Mol Sci 22:771. https://doi.org/10.3390/ ijms22020771.

43. Chomczynski P. 1993. A reagent for the single-step simultaneous isolation of RNA, DNA and proteins from cell and tissue samples. Biotechniques 15: 532-534.

44. Mayer FL, Wilson D, Hube B. 2013. Candida albicans pathogenicity mechanisms. Virulence 4:119-128. https://doi.org/10.4161/viru.22913.

45. Höfs S, Mogavero S, Hube B. 2016. Interaction of Candida albicans with host cells: virulence factors, host defense, escape strategies, and the microbiota. J Microbiol 54:149-169. https://doi.org/10.1007/s12275-016 $-5514-0$.

46. Araújo D, Henriques M, Silva S. 2017. Portrait of Candida species biofilm regulatory network genes. Trends Microbiol 25:62-75. https://doi.org/10 .1016/j.tim.2016.09.004.

47. Fourie R, Kuloyo OO, Mochochoko BM, Albertyn J, Pohl CH. 2018. Iron at the centre of Candida albicans interactions. Front Cell Infect Microbiol 8: 185. https://doi.org/10.3389/fcimb.2018.00185.

48. Edgar R, Domrachev M, Lash AE. 2002. Gene Expression Omnibus: NCBI gene expression and hybridization array data repository. Nucleic Acids Res 30:207-210. https://doi.org/10.1093/nar/30.1.207. 